\title{
Preference-based evolutionary algorithm for airport runway scheduling and ground movement optimisation
}

\author{
Michal Weiszer \\ School of Engineering \\ University of Lincoln \\ Lincoln, United Kingdom \\ Email: mweiszer@lincoln.ac.uk
}

\author{
Jun Chen \\ School of Engineering \\ University of Lincoln \\ Lincoln, United Kingdom \\ Email: juchen@lincoln.ac.uk
}

\author{
Paul Stewart \\ Institute for Innovation \\ in Sustainable Engineering \\ Derby, United Kingdom \\ Email: p.stewart1@derby.ac.uk
}

\begin{abstract}
As airports all over the world are becoming more congested together with stricter environmental regulations put in place, research on optimisation of airport surface operations started to consider both time and fuel related objectives. However, as both time and fuel can have a monetary cost associated with them, this information can be utilised as preference during the optimisation to guide the search process to a region with the most cost efficient solutions. In this paper, we solve the integrated optimisation problem combining runway scheduling and ground movement problem by using a multi-objective evolutionary framework. The proposed evolutionary algorithm is based on modified crowding distance and outranking relation which considers cost of delay and price of fuel. Moreover, the preferences are expressed in a such way, that they define a certain range in prices reflecting uncertainty. The preliminary results of computational experiments with data from a major airport show the efficiency of the proposed approach.
\end{abstract}

\section{INTRODUCTION}

With continuous growth of air traffic, which is predicted to carry double passengers in 2030 compared to 2013 [1], it is forecasted that without any action taken, many airports will become congested and air transportation as a whole will have a significant impact on the environment. As a result, research on management of aircraft movements on and in the proximity of airports attracted a lot of attention in general (e.g. [2], [3]) and airport surface operations in particular [4]. Specifically, the most research focused on optimisation of individual airport surface operations such as ground movement, runway scheduling or gate assignment to maximise the utilisation of available resources.

The objective of runway scheduling problem is often expressed as a minimisation of delay, the number of changes compared to First-come-first-served (FCFS) sequence, makespan or their combination. A wide range of exact and heuristic methods has been employed to solve this problem including dynamic programming [5], hybrid tabu search [6], and genetic algorithms [7]. A detailed review of recent research on runway scheduling problem can be found in [8].

The objective of ground movement has been considered to be mainly the minimisation of the total taxi time or other time related objectives [4]. Approaches to solve the ground movement problem include integer programming [9], [10] or a graph-based approach utilised in [11], [12]. In addition to the total taxi time, a few researchers started to consider also fuel consumption during ground movement as an objective of the multi-objective optimisation problem [13]. Since runway scheduling and ground movement are interconnected problems, recently both problems have been integrated together, minimising time related objective [14]-[16] or both time and fuel consumption [17] in a multi-objective manner.

As some of the above-mentioned approaches use multiobjective optimisation to minimise distinct objectives such as time and fuel at the same time, the result of such approach is a set of non-dominated solutions, from which the decision maker (DM) has to implement one solution. In practice, often is the case that there exist some preferences beforehand, such as the price of fuel or cost of a single minute of delay. However, as argued in [18], utilising this information to convert the multi-objective problem into a single-objective one is contraproductive. By finding only a single solution, DM is unable to investigate the properties of optimal/near-optimal solutions respecting higher-level preference information. Instead, this preference can be utilised during the optimisation to guide the search to an preferred region of interest (RoI) on the Pareto front. Research on incorporating preferences into multiobjective optimisation, whether in a-posteriori manner when the preferences are known before the search or in an interactive approach when preference information is iteratively obtained from the DM during the search, has been very active in the last years. Preferences are often expressed as reference points, goal vectors or aspiration levels corresponding to desired levels of objective values. The dominance relation is modified according to the distance to the reference point in [19] or aspiration level satisfaction in [20]. An achievement scalarizing function taking into account reference point is used to prefer some solutions closer to RoI in [21]-[24]. The original optimisation problem is modified by a weight distribution function in [25]. A binary preference in terms of linguistic variables is used in [26]; the crowding distance in Nondominated Sorting Genetic Algorithm-II (NSGA-II) [27] is changed in order to incorporate reference direction (weights) [28] or reference point [18], [23].

However, the desired values of objectives expressed as a reference point or aspiration levels are often unknown to the DM before the optimisation. As the result, an initial run of the optimisation algorithm is necessary to discover the whole 
Pareto front from which the DM can subsequently choose a RoI. On the other hand, if the preferences are modelled as weights, the extent of RoI is usually defined by some nonintuitive parameter during the search as in [28].

In this paper, we solve the integrated optimisation problem combining runway scheduling and ground movement problem, similar as in [17], by using a multi-objective evolutionary optimisation (EMO) framework based on genetic algorithm (GA) and NSGA-II. The proposed algorithm employs a modified crowding distance presented in [23] and outranking relation of the Light Beam Search (LBS) method [29], which considers preference information in terms of cost of delay and price of fuel, guiding the search towards the most cost optimal RoI. Moreover, the preferences are expressed in a such way, that they give the DM a possibility to define a certain range of prices reflecting uncertainty.

The rest of the paper is organised as follows. Section II provides details about individual components of the integrated model, including runway scheduling and ground movement problem. The proposed EMO framework taking into account preferences is described in Section III A set of computational experiments is carried out using data instances from Doha International Airport in Section IV. Finally, conclusions are drawn in Section $\mathrm{V}$

\section{PROBLEM DESCRIPTION}

This section provides a description of the integrated optimisation problem of airport ground operations combining the runway scheduling and ground movement problem similar to one introduced in [17].

\section{A. Runway scheduling problem}

The aim of runway scheduling in this paper is to find the optimal landing/take-off time of arriving/departing aircraft at the given runway minimising the delay and fuel burnt during waiting while respecting given safety constraints. In this paper, only take-off times are optimised whereas landing times are considered to be fixed, as from the practical point of view, it is easier to control taking-off aircraft still on the ground rather than airborne arriving aircraft.

The main constraint that limits the throughput of the runway is the minimum time interval between landing/takingoff aircraft. These minimum separations are due to wake vortices created by moving aircraft and in-flight separation constraints. In this paper, only separation due wake vortices is taken into account. The strength of wake vortices and thus separation depends on the aircraft type and is approximately proportional to its weight.

Let $M=(A \cup D)$ be the set of total $|M|=m$ arriving aircraft $A$ and departing aircraft $D$. Let $V\left(v_{i}, v_{j}\right)$ be the function to calculate the wake vortex separations from weight categories $v_{i}$ and $v_{j}$ of leading aircraft $i$ and trailing aircraft $j$. The wake vortex separations used in this paper satisfy the triangle inequality $V\left(v_{i}, v_{j}\right)+V\left(v_{j}, v_{e}\right) \geq V\left(v_{i}, v_{e}\right)$ for aircraft taking off in the order $i, j, e$ and are given in Table I

Let $r_{i}$ be the actual landing time for aircraft $i \in A$ and takeoff time for aircraft $i \in D$. For arriving aircraft, $r_{i}$ is given. For departing aircraft, let $d_{i}$ denote the time the departing
TABLE I: Separations in seconds between departing (D) and arriving (A) flights for weight classes: Heavy (H), Large (L), Small (S).

\begin{tabular}{cccccccc}
\hline \multirow{6}{*}{ Leading } & & \multicolumn{7}{c}{ Trailing } \\
\cline { 3 - 8 } & & A-H & A-L & A-S & D-H & D-L & D-S \\
& A-H & 96 & 157 & 207 & 60 & 60 & 60 \\
& A-L & 60 & 69 & 123 & 60 & 60 & 60 \\
& A-S & 60 & 69 & 82 & 60 & 60 & 60 \\
& D-H & 60 & 60 & 60 & 96 & 120 & 120 \\
& D-L & 60 & 60 & 60 & 60 & 60 & 60 \\
& D-S & 60 & 60 & 60 & 60 & 60 & 60 \\
\hline
\end{tabular}

aircraft $i \in D$ arrived at the runway holding point. Aircraft $i \in D$ can take-off immediately, i.e. $d_{i}=r_{i}$ if there is enough time elapsed from landing/take-off time $r_{i-1}$ of the previous aircraft $i-1$ to comply with separation given by $V\left(v_{i}, v_{i-1}\right)$, otherwise, the departing aircraft $i$ has to wait at the runway holding point until it is safe to take-off:

$r_{i}= \begin{cases}d_{i} & \text { if } d_{i}-r_{i-1} \geq V\left(v_{i}, v_{i-1}\right), \\ d_{i}+V\left(v_{i}, v_{i-1}\right)-\left(d_{i}-r_{i-1}\right) & \text { otherwise. }\end{cases}$

Then, the waiting time $w_{i}$ of the departing aircraft $i \in D$ is equal to $w_{i}=d_{i}-r_{i}$.

The objective of the runway scheduling is to minimise the total runway delay $t^{r w y}$ and the total runway fuel $f^{r w y}$ burned by aircraft while waiting to take-off which depends on the delay $w_{i}$ and idle fuel flow $\phi_{v_{i}}$ specified for the weight category $v_{i}$ :

$$
\begin{gathered}
t^{r w y}=\sum_{i=1}^{D} w_{i}, \\
f^{r w y}=\sum_{i=1}^{D} w_{i} \cdot \phi_{v_{i}} .
\end{gathered}
$$

The idle fuel flow $\phi_{v_{i}}$ corresponds to fuel flow from the International Civil Aviation Organization (ICAO) engine database for $5 \%$ of full power thrust of the representative aircraft, as explained in Section II-B.

\section{B. Ground movement problem}

The aim of the ground movement problem is to find routes and schedules for aircraft taxiing from runway to gate/stand and vice versa in a time and fuel efficient manner, respecting routes of other aircraft while preventing conflicts between them.

In this paper, due to the simple layout of the airport, the problem of finding routes is reduced to the shortest path problem. The shortest paths between each gate/stand and runway exit points are pre-calculated beforehand. Then, for each path optimised speed profiles are found by a specialized heuristic described in [30]. The speed profiles are optimised in a multi-objective manner, minimising taxi time and fuel consumption for the given route. The resulting non-dominated speed profiles are stored in a look-up table, with a separate table for each weight category $v_{i}$ and then retrieved during the on-line optimisation in order to save computational time. 
Given the route of the aircraft and time needed to travel from origin to destination depends on the chosen speed profile, some delay may be added in order to prevent conflicts between taxiing aircraft. The conflicts between aircraft are solved by adding a small buffer time such that aircraft always maintain a safe time distance $\delta=12 \mathrm{~s}$ between them (which corresponds to approximately $62 \mathrm{~m}$ at taxiing speed $10 \mathrm{knots}$ ).

Let $y_{i}$ be an integer representing the speed profile of aircraft $i$ from the Pareto front of efficient speed profiles retrieved from the look-up table for the shortest route $q_{i}$ from the runway to the stand $s_{i}$ for arriving aircraft $i \in A$, or vice versa for departing aircraft $i \in D$. We define a function $T\left(q_{i}, y_{i}\right)$ which returns travel time of aircraft $i$ taxiing on route $q_{i}$ for the given speed profile $y_{i}$, including a delay to prevent taxiing conflicts.

In order to retrieve the fuel consumption of aircraft $i$ of weight category $v_{i}$, for route $q_{i}$, using speed profile $y_{i}$, a function $F\left(q_{i}, y_{i}, v_{i}\right)$ is defined. The function $F\left(q_{i}, y_{i}, v_{i}\right)$ is based on the method introduced in [30], which employs physics-based equations taking into account the acceleration force and rolling resistance to calculate thrust. The calculated thrust and aircraft engines are then mapped into corresponding fuel flows according to the ICAO emissions database.

Then, the objective of the ground movement problem is to minimise the total taxi time $t^{t a x i}$ and the total fuel $f^{t a x i}$ burned during ground movement:

$$
\begin{aligned}
t^{\text {taxi }} & =\sum_{i=1}^{M} T\left(y_{i}\right), \\
f^{\text {taxi }} & =\sum_{i=1}^{M} F\left(y_{i}\right) .
\end{aligned}
$$

\section{Integrated optimisation problem}

The runway scheduling and ground movement problem are combined into the integrated bi-objective optimisation problem with the following objective functions:

$$
\begin{aligned}
& \min g_{1}=t^{t a x i}+t^{r w y}, \\
& \min g_{2}=f^{t a x i}+f^{r w y},
\end{aligned}
$$

where $g_{1}$ corresponds to the total time and $g_{2}$ is the fuel consumption to be minimised. The decision variables for this optimisation problem are the pushback time for departing aircraft $x_{i} \in\{-300, \ldots, 300\}$ and the speed profile $y_{i} \in$ $\{1, \ldots, 12\}$ for all aircraft. The pushback time $x_{i}$ represents an integer number of seconds before/after the baseline time given as input flight schedule when the aircraft starts taxiing. The value of speed profile $y_{i}$ determines which solution from the pre-computed look-up table of non-dominated speed profiles is going to be selected, ranging from 1 representing the most time-efficient (fastest) speed profile to 12 standing for the most fuel efficient one. The number of speed profiles depends on the heuristic used for their generation, for details see [30].

Given the decision variables, the objective function values $g_{1}, g_{2}$ are determined as follows. Firstly, aircraft are considered sequentially according to their initial sequence specified by the input flight schedule. For each arriving aircraft $i \in A$ a shortest route $q_{i}$ is retrieved from the look-up table between the runway and designated gate/stand $s_{i}$ or in the opposite direction for departing aircraft $i \in D$. The route $q_{i}$, weight category $v_{i}$ and speed profile value $y_{i}$ determine which precomputed speed profile is retrieved from the look-up table and used to schedule aircraft $i$ along the route $q_{i}$ after all taxiing conflicts have been resolved. Then, the total taxi time $t^{\text {taxi }}$ and the total fuel $f^{\text {taxi }}$ are computed as stated in Section II-B Based on taxi time $T\left(y_{i}\right)$, the runway holding point arrival time $d_{i}$ is determined for each departing aircraft $i \in D$. The delay $w_{i}$ is calculated as defined in section II-A and summed for all departing aircraft $i \in D$ to get the total runway delay $t^{r w y}$ and subsequently the total fuel $f^{r w y}$. Finally, objectives $g_{1}, g_{2}$ are calculated according to 5 and 6 .

\section{Preferences based on costs}

The bi-objective optimisation problem formulated in Section II-C minimises two objective functions $g_{1}, g_{2}$ simultaneously. In practice, the total time $g_{1}$ and fuel consumption $g_{2}$ are associated with economic costs incurred by operations on the airport surface. For example, the fuel consumption $g_{2}$ is connected to fuel cost defined by fuel price. Similarly, total time $g_{1}$ incurs costs due to the fact, that each second of delay (i.e. unproductive time spent taxiing or waiting at runway) is an extra cost in terms of crew, maintenance, deprecation of aircraft, etc. Therefore, once costs related to $g_{1}, g_{2}$ are known, they can be used as weights during the optimisation, directing the search to a single cost-optimal solution. However, determining these cost, specifically cost of delay, is often difficult and only approximate as for example, crew wages or maintenance fees vary from airline to airline. Therefore, it might be more useful to guide the search to a region on the Pareto front containing solutions optimal for a certain cost range. We define a price vector $\mathbf{c}=\left[c_{1}, \ldots, c_{n}\right]$ which, in general, specifies cost for each one of $n$ objectives. Furthermore, to include the uncertainty in costs, $l$ boundary price vectors $\mathbf{c}^{B_{l}}=\left[c_{1}^{B_{l}}, \ldots, c_{n}^{B_{l}}\right]$ are defined. The number of boundary vectors depends on the DM. For example, DM can specify only upper bound for cost, both upper and lower bounds or different combinations in case of many objectives.

Then, each solution $z_{j}$ found during optimisation can be evaluated by function $C^{\text {total }}\left(\mathbf{z}_{j}, \mathbf{c}\right)$ in terms of total monetary cost, using price vector c:

$$
C^{\text {total }}\left(\mathbf{z}_{j}, \mathbf{c}\right)=\sum_{k=1}^{n} c_{k} \cdot g_{k}\left(\mathbf{z}_{j}\right) .
$$

\section{EMO FRAMEWORK WITH PREFERENCES}

In order to optimise the objective functions $g_{1}, g_{2}$ of the integrated optimisation problem stated in Section II taking into account preferences given by costs, the following EMO framework is proposed in this section. Firstly, the DM is asked to provide a price vector $\mathbf{c}$ and $l$ boundary price vectors $\mathbf{c}^{B_{l}}$ as defined in Section II-D Then, the search is divided into two parts:

1) A single objective optimisation search with aggregated objective function is performed by $\mathrm{GA}$ to quickly approach to the vicinity of the global Pareto front. 
2) The best solutions from the single objective optimisation part are seeded into a preference-based EMO algorithm to reach RoI of the global Pareto front.

The single-objective optimisation is carried out by the GA minimising total costs $C^{\text {total }}$ as defined in 7 The singleobjective search is further accelerated by taking advantage of known preferences during the search process. For each aircraft $i$, speed profile is fixed to a value $y_{i} \in\{1,12\}$ with minimum $C^{\text {total }}$. As a result, the search space is restricted to decision variable $x_{i}$ promising faster convergence, however sacrificing some of the optimality.

\section{A. Preference-based NSGA-II}

For the second phase, the EMO algorithm proposed in this paper, denoted as Preference-based NSGA-II (P-NSGAII), is based on Light Beam Search EMO algorithm [23] which is a derivative of NSGA-II [27]. The initial population of random solutions is seeded with a specified number of best solutions (set to 10 in this paper) found by GA during the first phase. In the second phase, P-NSGA-II considers all decision variables $x_{i}$ and $y_{i}$ thus finding potentially better solutions. The P-NSGA-II performs the usual EMO operations: selection, crossover, mutation, replacement for the specified number of generations similar to original NSGA-II. In order to incorporate preferences into EMO algorithm, the replacement procedure which selects the solutions surviving to the next generation is modified. The original replacement procedure in NSGA-II favours non-dominated solutions with large crowding distance. In P-NSGA-II, the replacement is carried out with modified crowding distance to prefer solutions closer to RoI.

During replacement procedure, non-domination ranking is carried out for all solutions in the population. Then, the middle point and characteristic neighbours are determined as depicted on Fig. 1. The middle point $\mathbf{z}^{C}$ is a solution $\mathbf{z}_{j}$ with minimum cost defined by price vector $\mathbf{c}$ :

$$
\mathbf{z}^{C}=\underset{\mathbf{z}_{j}}{\arg \min }\left(C^{\text {total }}\left(\mathbf{z}_{j}, \mathbf{c}\right)\right) .
$$

The characteristic neighbour $\mathbf{z}^{B_{l}}$ is a solution $\mathbf{z}_{j}$ for which the cost using the boundary price vector $\mathbf{c}^{B_{l}}$ is minimum:

$$
\mathbf{z}^{B_{l}}=\underset{\mathbf{z}_{j}}{\arg \min }\left(C^{\text {total }}\left(\mathbf{z}_{j}, \mathbf{c}^{B_{l}}\right)\right) \text {. }
$$

Subsequently, for each solution $\mathbf{z}_{j}$, belonging to the same non-dominated front, crowding distance $c d_{j}$ is calculated:

$$
c d_{j}= \begin{cases}\infty & \text { if } \mathbf{z}_{j} \text { is middle point } \mathbf{z}^{C}, \\ M+d_{j} & \text { else if } \mathbf{z}_{\mathbf{j}} \text { outranks } \mathbf{z}^{C}, \\ 1 / C^{\text {total }}\left(\mathbf{z}_{j}, \mathbf{c}\right) & \text { otherwise. }\end{cases}
$$

If the solution $\mathbf{z}_{j}$ is the middle point, it is assigned an infinite crowding distance, so it is always included in the next generation. Other solutions are categorized according to their outranking relation to $\mathbf{z}^{C}$. A solution $\mathbf{z}_{j}$ is said to outrank middle point $\mathbf{z}^{C}$ (denoted as $\mathbf{z}_{j} S \mathbf{z}^{C}$ ) if $m_{v}\left(\mathbf{z}_{C}, \mathbf{z}_{X}\right)=0$, where $m_{v}$ is defined as follows:

$m_{v}\left(\mathbf{z}^{C}, \mathbf{z}_{X}\right)=\operatorname{card}\left\{k: g_{k}\left(\mathbf{z}_{X}\right)-g_{k}\left(\mathbf{z}^{C}\right) \geq v_{k}, k=1, \ldots, n\right\}$

Solutions outranking the middle point $\mathbf{z}^{C}$ are considered to be as good as $\mathbf{z}^{C}$ and form the outranking neighbourhood. As

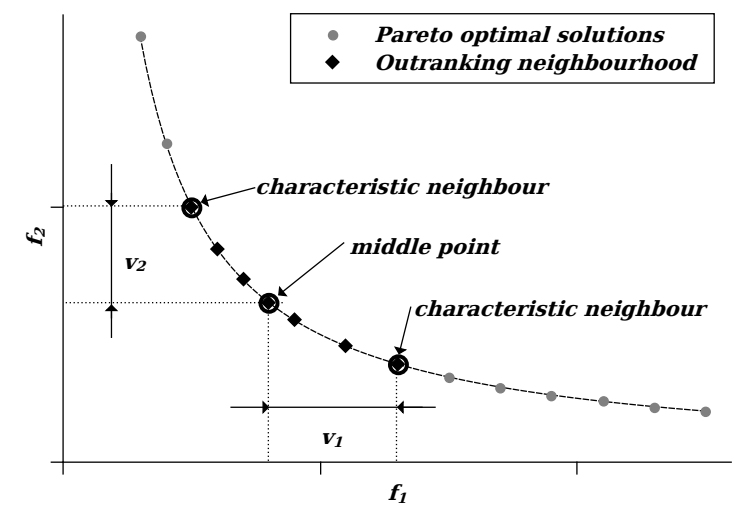

Fig. 1: Preferred region on Pareto front.

all solutions in outranking neighbourhood belong to the same non-dominated front, if solution $\mathbf{z}_{j}$ is better than $\mathbf{z}^{C}$ in some objectives, then it must be worse in at least one other objective. Solution $\mathbf{z}_{j} S \mathbf{z}^{C}$ if this deterioration in objective $k$ is not larger than veto threshold $v_{k}$. The veto threshold $v_{k}$ for objective $k$ is determined by characteristic neighbours:

$$
v_{k}=\max \left\{g_{k}\left(\mathbf{z}^{B_{l}}\right), l=1, \ldots, n\right\} .
$$

Solutions $\mathbf{z}_{j}$ in the outranking neighbourhood are assigned $M+d_{j}$ crowding distance, where $M$ is a big positive integer, set to $M=10^{6}$ in this work and $d_{j}$ is the original crowding distance as defined by Deb [27]:

$$
d_{j}=\sum_{k=1}^{K} \frac{g_{k}\left(z_{j+1, k}\right)-g_{k}\left(z_{j-1, k}\right)}{g_{k}^{\max }-g_{k}^{\min }}
$$

Where $g_{k}^{\max }$ is the maximum and $g_{k}^{\min }$ the minimum value of the objective function $g_{k}$ found so far. The big integer $M$ ensures that crowding distance $c d_{j}$ is large enough to guarantee inclusion into the next generation whereas $d_{j}$ causes the preference of solutions in less crowded areas, resulting in a uniformly distributed solutions across the characteristic neighbourhood.

For remaining solutions, the crowding distance $c d_{j}$ is inversely related to $C^{\text {total }}\left(\mathbf{z}_{j}, \mathbf{c}\right)$. As a result, solutions $\mathbf{z}_{j}$ with smaller $C^{\text {total }}\left(\mathbf{z}_{j}, \mathbf{c}\right)$ and thus closer to the RoI are assigned larger crowding distance giving them larger chance to be included in the next generation.

\section{COMputational RESUlts AND Discussion}

\section{A. Experimental setup}

The proposed EMO framework was tested on a dataset of real arrival and departure flights on Doha International Airport (DOH) which was the largest airport in Qatar and a hub airport for Qatar Airways until the new Hamad International airport was completed in late April 2014. DOH airport has one runway and 55 stands. The data were recorded on 16th March 2014 and divided into two instances doh 1 representing medium traffic conditions and doh 2 for high traffic conditions. The instance doh1 includes 96 flights between 17:00 and 21:00 UTC from which 50 are arrivals and 46 departures. The instance doh2 
TABLE II: Specifications of the representative aircraft.

\begin{tabular}{lrrr}
\hline & Learjet 35A & Airbus A320 & Airbus A333 \\
\hline Take-off weight & $8300 \mathrm{~kg}$ & $78000 \mathrm{~kg}$ & $230000 \mathrm{~kg}$ \\
Engines & TFE731-2-2B & CMF56-5-A1 & CF6-80E1A2 \\
Number of engines & 2 & 2 & 2 \\
Rated output $R$ & $2 \times 15.6 \mathrm{kN}$ & $2 \times 111.2 \mathrm{kN}$ & $2 \times 287 \mathrm{kN}$ \\
Rolling resistance & $1221 \mathrm{~N}$ & $11.48 \mathrm{kN}$ & $33.84 \mathrm{kN}$ \\
Fuel flow at $7 \% R$ & $0.024 \mathrm{~kg} \cdot \mathrm{s}^{-1}$ & $0.101 \mathrm{~kg} \cdot \mathrm{s}^{-1}$ & $0.228 \mathrm{~kg} \cdot \mathrm{s}^{-1}$ \\
Fuel flow at $30 \% R$ & $0.067 \mathrm{~kg} \cdot \mathrm{s}^{-1}$ & $0.291 \mathrm{~kg} \cdot \mathrm{s}^{-1}$ & $0.724 \mathrm{~kg} \cdot \mathrm{s}^{-1}$ \\
\hline
\end{tabular}

TABLE III: Average $I_{\epsilon}$ indicator for 30 runs.

\begin{tabular}{lccc}
\hline & NSGA-II & P-NSGA-II & P-NSGA-II with seed \\
\hline doh 1 & 1.061 & 1.053 & 1.016 \\
doh 2 & 1.219 & 1.207 & 1.040 \\
\hline
\end{tabular}

consists of 84 flights between 21:00 and 23:00 UTC from which there are 27 arrivals and 57 departures. The data provided specified landing/pushback times and gates/runway exits for each flight.

The aircraft have been divided into 3 groups according their wake vortex separation requirements as defined in [31]. For each category, a representative aircraft is designated and its specifications are used during the fuel consumption calculation. The specifications are summarized in Table II

The computational experiments were performed on a computer with an Intel i3-2120 processor and 3.16 GB of RAM, running Linux. The EMO framework is implemented using the Inspyred package for Python [32]. The termination criteria for EMO framework was set to 900 seconds, of which 600 seconds is allocated to GA and 300 seconds to P-NSGA-II. Based on initial experiments, the number of individuals in population for GA was set to 200 and 50 for P-NSGA-II. The price vector $\mathbf{c}$ is equal to $[0.469,0.71]$, where the first element corresponds to costs of delay as defined in [17] and the second element is the jet fuel price (as of 14.1.2014), both in Euro. The boundary vector $\mathbf{c}^{B_{1}}=[1.2 \times 0.469,0.8 \times 0.71]$ and $\mathbf{c}^{B_{2}}[0.8 \times 0.469,1.2 \times 0.71]$.

\section{B. Computational results}

The performance of the proposed two-phase EMO framework (P-NSGA-II with seed) was compared to original NSGAII and P-NSGA-II without the seed from single-objective GA. Fig. 2 shows the best Pareto fronts from 30 runs of the algorithms for doh 1 instance. As can be seen, singleobjective GA was able to find better solutions than NSGAII and comparable with P-NSGA-II alone. Then, P-NSGAII could further improve the solutions from the initial seed, focusing the search on RoI.

The multiplicative unary epsilon indicator $I_{\epsilon}$ is used as the performance index, expressing the distance of the resulting Pareto front to the global Pareto front, which was constructed by considering all solutions found during the experiments and leaving only non-dominated ones. Average $I_{\epsilon}$ indicator values for 30 runs of the algorithms are given in Table III]

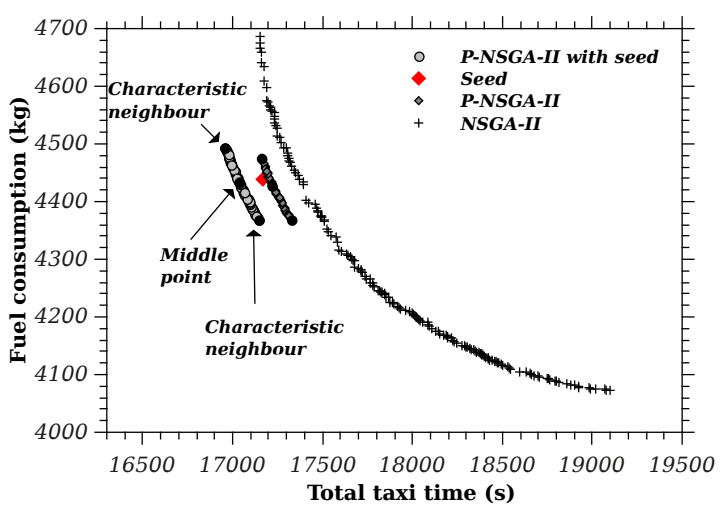

Fig. 2: Pareto fronts for doh1.

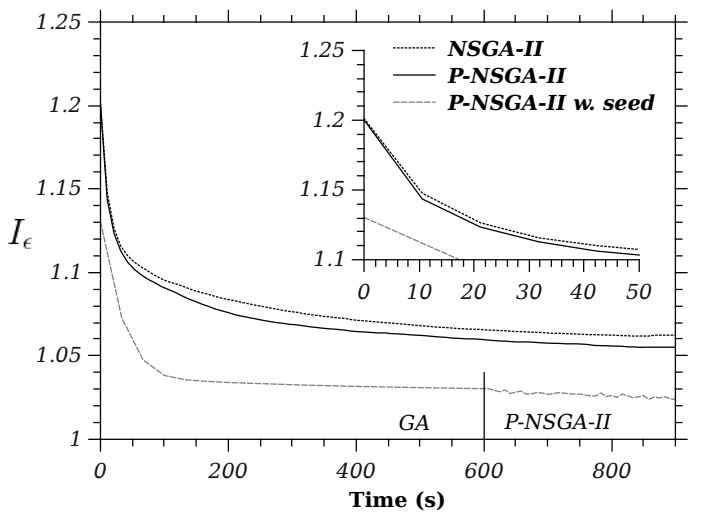

Fig. 3: Averaged convergence for 30 runs for doh 1 instance.

The results of $I_{\epsilon}$ show that P-NSGA-II alone could only marginally improve the performance compared to NSGAII. However, adding seed to P-NSGA-II significantly helped the algorithm to find better solutions. The improving effect is even more evident for doh2 instance with high traffic conditions. We can hypothesize that beneficial impact of initial single-objective GA on the overall performance of the EMO framework will increase in case of many (i.e. more than 3) objectives as indicated in [22], due to the fact that many objectives resulting in high number of non-dominated solutions deteriorate the performance of EMO algorithms.

The convergence during the evolution for the doh 1 is documented in Fig. 3 Single-objective GA in the first phase of the search quickly finds good solutions, helping the P-NSGAII in the second phase obtain good values of $I_{\epsilon}$. Furthermore, fixing speed profiles $y_{i}$ for GA results in better values of $I_{\epsilon}$ for the initial population, effectively accelerating the search.

\section{CONClusion}

In this paper, a multi-objective evolutionary framework taking into account preferences in an a-priori manner is proposed to solve integrated optimisation problem of runway scheduling and ground movement on airport surface. The preference was incorporated in the first phase to transform the original multiobjective problem to a single-objective one to quickly approach 
the region of interest. Then, in the second phase a modified crowding distance in NSGA-II was applied to finally arrive to Pareto front of cost-efficient solutions. The preliminary computational experiments conducted on real-world data from a major Asian airport showed promising results and indicate that the proposed approach could be suitable as an optimisation framework for decision support at the airport. The evolutionary framework utilising the preferences given as costs associated with objectives successfully accelerated the search. Moreover, the input values of costs enabled to control the extent of the resulting region and include some uncertainty about the prices. The incorporation of preferences helps the decision maker to focus his attention on only cost-effective solutions in a shorter computational time. For the future research, the approach needs to be validated on a more diverse set of problem instances. Also, the idea of including uncertainty into preference information deserves more attention. As an example, the uncertainty could be expressed in terms of fuzzy values.

\section{REFERENCES}

[1] ICAO. (2014) Annual Report of the ICAO Council: 2013 The World of Air Transport. [Online]. Available: http://www.icao.int/ annual-report-2013/Pages/the-world-of-air-transport-in-2013.aspx

[2] L. Bianco, P. Dell'Olmo, and S. Giordani, "Scheduling models for air traffic control in terminal areas," Journal of Scheduling, vol. 9, no. 3, pp. 223-253, 2006. [Online]. Available: http: //dx.doi.org/10.1007/s10951-006-6779-7

[3] M. Samà, A. D'Ariano, and D. Pacciarelli, "Rolling horizon approach for aircraft scheduling in the terminal control area of busy airports," Transportation Research Part E: Logistics and Transportation Review, vol. 60, no. 0, pp. 140-155, 2013. [Online]. Available: http://www.sciencedirect.com/science/article/pii/S136655451300118X

[4] J. A. Atkin, E. K. Burke, and S. Ravizza, "The airport ground movement problem: Past and current research and future directions," Proceedings of the 4th International Conference on Research in Air Transportation (ICRAT), Budapest, Hungary, pp. 131-138, 2010.

[5] H. Balakrishnan and B. Chandran, "Scheduling aircraft landings under constrained position shifting," in AIAA Guidance, Navigation, and Control Conference and Exhibit, Keystone, CO, 2006.

[6] J. A. D. Atkin, E. K. Burke, J. S. Greenwood, and D. Reeson, "Hybrid Metaheuristics to Aid Runway Scheduling at London Heathrow Airport," Transportation Science, vol. 41, no. 1, pp. 90-106, 2007.

[7] X.-B. Hu and E. Di Paolo, "Binary-Representation-Based Genetic Algorithm for Aircraft Arrival Sequencing and Scheduling," Intelligent Transportation Systems, IEEE Transactions on, vol. 9, no. 2, pp. 301310, June 2008.

[8] J. Bennell, M. Mesgarpour, and C. Potts, "Airport runway scheduling," Annals of Operations Research, vol. 204, no. 1, pp. 249-270, 2013 [Online]. Available: http://dx.doi.org/10.1007/s10479-012-1268-1

[9] A. Marín and E. Codina, "Network design: taxi planning," Annals of Operations Research, vol. 157, no. 1, pp. 135-151, 2008.

[10] P. C. Roling and H. G. Visser, "Optimal Airport Surface Traffic Planning Using Mixed-Integer Linear Programming," International Journal of Aerospace Engineering, vol. vol. 2008, p. 11, 2008.

[11] S. Ravizza, J. A. Atkin, and E. K. Burke, "A more realistic approach for airport ground movement optimisation with stand holding," Journal of Scheduling, pp. 1-14, 2013.

[12] C. Lesire, "An Iterative A* Algorithm for Planning of Airport Ground Movements," 19th European Conference on Artificial Intelligence (ECAI)/6th Conference on Prestigious Applications of Intelligent Systems (PAIS), Lisbon, Portugal, August 16-20, 2010.

[13] S. Ravizza, J. Chen, J. A. Atkin, E. K. Burke, and P. Stewart, "The trade-off between taxi time and fuel consumption in airport ground movement," Public Transport, vol. 5, no. 1-2, pp. 25-40, 2013.
[14] R. Deau, J. Gotteland, and N. Durand, "Airport surface management and runways scheduling," in Proceedings of the 8th USA/Europe Air Traffic Management Research and Development Seminar, Napa, CA, USA, 2009

[15] G. Clare and A. Richards, "Optimization of Taxiway Routing and Runway Scheduling," Intelligent Transportation Systems, IEEE Transactions on, vol. 12, no. 4, pp. 1000-1013, Dec 2011.

[16] M. Frankovich and D. Bertsimas, "Air Traffic Flow Management at Airports: A Unified Optimization Approach," in Tenth USA/EUROPE Air Traffic Management Research \& Development Seminar, June 2013.

[17] M. Weiszer, J. Chen, and G. Locatelli, "An integrated optimisation approach to airport ground operations to foster sustainability in the aviation sector," Applied Energy, 2015, in press. [Online]. Available: http://dx.doi.org/10.1016/j.apenergy.2015.04.039.

[18] K. Deb, J. Sundar, N. Udaya Bhaskara Rao, and S. Chaudhuri, "Reference point based multi-objective optimization using evolutionary algorithms," International Journal of Computational Intelligence Research vol. 2, no. 3, pp. 273-286, 2006.

[19] L. Ben Said, S. Bechikh, and K. Ghédira, "The r-dominance: a new dominance relation for interactive evolutionary multicriteria decision making," Evolutionary Computation, IEEE Transactions on, vol. 14, no. 5, pp. 801-818, 2010.

[20] J. Molina, L. V. Santana, A. G. Hernández-Díaz, C. A. Coello Coello, and R. Caballero, "g-dominance: Reference point based dominance for multiobjective metaheuristics," European Journal of Operational Research, vol. 197, no. 2, pp. 685-692, 2009.

[21] L. Thiele, K. Miettinen, P. J. Korhonen, and J. Molina, "A preferencebased evolutionary algorithm for multi-objective optimization," Evolutionary Computation, vol. 17, no. 3, pp. 411-436, 2009.

[22] A. López-Jaimes and C. A. C. Coello, "Including preferences into a multiobjective evolutionary algorithm to deal with manyobjective engineering optimization problems," Information Sciences, vol. 277, no. 0, pp. 1-20, 2014. [Online]. Available: http: //www.sciencedirect.com/science/article/pii/S0020025514004708

[23] K. Deb and A. Kumar, "Light beam search based multi-objective optimization using evolutionary algorithms," in Evolutionary Computation, 2007. CEC 2007. IEEE Congress on. IEEE, 2007, pp. 2125-2132.

[24] S. Huber, M. J. Geiger, M. Sevaux et al., "Interactive approach to the Inventory Routing Problem: computational speedup through focused search," in Proceedings of the Logistikmanagement 2013 conference, 2013.

[25] T. Friedrich, T. Kroeger, and F. Neumann, "Weighted preferences in evolutionary multi-objective optimization," in AI 2011: Advances in Artificial Intelligence. Springer, 2011, pp. 291-300.

[26] D. Cvetkovic and I. Parmee, "Preferences and their application in evolutionary multiobjective optimization," Evolutionary Computation, IEEE Transactions on, vol. 6, no. 1, pp. 42-57, Feb 2002.

[27] K. Deb, A. Pratap, S. Agarwal, and T. Meyarivan, "A fast and elitist multiobjective genetic algorithm: NSGA-II," Evolutionary Computation, IEEE Transactions on, vol. 6, no. 2, pp. 182-197, Apr. 2002.

[28] J. Branke and K. Deb, "Integrating user preferences into evolutionary multi-objective optimization," in Knowledge incorporation in evolutionary computation. Springer, 2005, pp. 461-477.

[29] A. Jaszkiewicz and R. Słowiński, "The 'Light Beam Search'approachan overview of methodology applications," European Journal of Operational Research, vol. 113, no. 2, pp. 300-314, 1999.

[30] M. Weiszer, J. Chen, S. Ravizza, J. Atkin, and P. Stewart, "A heuristic approach to greener airport ground movement," July 2014, pp. 32803286.

[31] ICAO, Air Traffic Management: Procedures for Air Navigation Services, 2007, 15th edition. Doc 4444 ATM/501.

[32] A. Garrett, "Inspyred," 2012, (Version 1.0) [software]. Inspired Intelligence Initiative. Retrieved from http://inspyred.github.com. 